\title{
Association between fibrinogen receptor (Glycoprotein llb) polymorphism and the risk of venous thromboembolism: a systematic review
}

\author{
Zahra Rezaei Dezaki ${ }^{1}$, Raihaneh Bagheri ${ }^{2}$ and Batoul Pourgheysari, ${ }^{3,4^{*}}$
}

\begin{abstract}
Background: The fibrinogen receptor is an integrin on the platelet surface and is shaped from two types of glycoprotein (GP) subunits, GPIIb and GPIIla. Membrane glycoprotein Ilb/llla plays an important role in platelet function. The gene encoding the glycoprotein Illa shows a common polymorphism, PLA2 that increases the binding of the receptor to fibrinogen and enhances the platelet aggregation. The clinical impact of PLA2 polymorphism has been studied in some diseases, but the definition of its exact role on venous thromboembolism complications has been challenging. The present systematic review aimed to clarify the association of PLA2 polymorphism and venous thromboembolism.

Main text: In this study, Electronic databases including PubMed, Embase, Scopus, Web of Science, and Cochrane Library were searched. All the assessed studies focused on the relationship between PLA2 polymorphism and venous thromboembolism. Five studies were eligible for systematic review. One study revealed a significant correlation between PLA2 polymorphism and venous thromboembolism. PLA2 polymorphism was associated with deep vein thrombosis in one study and pulmonary thromboembolism in another one.

Conclusion: The published data supported the hypothesis that having the PLA2 polymorphism of GPIIla may be a risk factor for venous thromboembolism, but the association cannot be concluded; it needs more clinical investigation.
\end{abstract}

Keywords: Venous thromboembolism, Deep vein thrombosis, Pulmonary embolism, PLA2 polymorphism

\section{Background}

The platelet fibrinogen receptor is essential for preliminary hemostasis, as it regulates platelet aggregation and binds to a combination of fibrinogen, von Willebrand factor (vWF), and fibronectin for the formation of a stable thrombus following vascular injury [1-3].

The mature fibrinogen receptor is an integrin on the platelet surface [4] and is formed from two types of glycoprotein (GP) subunits, GPIIb and GPIIIa [5]. The GP IIIa subunit is a great polymorphic protein with

\footnotetext{
*Correspondence: Zahra.rzi69@gmail.com; bat238@yahoo.com

${ }^{3}$ Medical Plants Research Center, Basic Health Sciences Institute, Shahrekord

University of Medical Sciences, Shahrekord, Iran

${ }^{4}$ Department of Pathology and Hematology, Shahrekord University of

Medical Sciences, Shahrekord, Iran

Full list of author information is available at the end of the article
}

platelet antigen 1 (PLA1) and 2 (PLA2) as the utmost stable allelic variants [6]. The PLA2 polymorphism of GP IIIa results from the substitution of leucine by proline at position 33 [7].

Previous studies demonstrated that the PLA2 isoform increases the binding of the GP IIb/IIIa receptor to fibrinogen and enhances the platelet aggregation, thus it is a possible factor for thrombotic tendency with other prevalent recognized inherited thrombophilic factors such as factor V Leiden (FVL), mutation G20210A in prothrombin gene, and factor XIII polymorphism [8-11].

The clinical impact of PLA2 polymorphism (formed from GP IIIa) has been associated with an increased risk of several diseases where thrombus formation is a crucial pathogenic factor for their development such as 
myocardial infarction and stroke, and was suggested as a risk factor for venous thromboembolism (VTE) [2, 1215]. VTE, including pulmonary embolism (PE) and deep vein thrombosis (DVT), is a major public health problem [16], mostly for hospitalized patients [17] and is among the utmost ordinary preventable reasons of hospital death [18]. VTE appears to be triggered by acquired and hereditary risk factors including mutations in genes encoding hemostatic proteins such as coagulation factors, coagulations inhibitors, and platelet glycoproteins.

Some studies investigated the role of the PLA2 polymorphism in DVT, PE, or VTE, but this is still controversial.

To the best of our knowledge, the association between PLA2 polymorphism and VTE has not been systematically assessed. Therefore, this systematic review aimed to evaluate the prognostic significance of PLA2 polymorphism in DVT, PE, and VTE patients to explore the relationship between this polymorphism and venous thrombotic events.

\section{Main text}

\section{Search strategy}

Search strategy and selection of articles for this systematic review were performed in accordance with the Preferred Reporting Items for Systematic Reviews and Meta-analyses (PRISMA) statement (Fig. 1).

We searched the different databases including Pub Med, Scopus, Web of Science, Embase, and Cochrane library databases up to January 2018. Electronic searches were performed combining some keywords that were planned to proper match to MeSH terms.

"PLA2 polymorphism" and "Deep vein thrombosis," "PLA2 polymorphism" and "Pulmonary embolism," "PLA2 polymorphism" and "Venous thromboembolism" or "VTE," "PLA2 polymorphism" and "Deep vein thrombosis" or "DVT," or "Pulmonary Embolism" or "Venous thromboembolism."

We also manually searched the reference lists of primary studies and reviews, in order to find the studies which cannot be found via an electronic search.

\section{Selection criteria}

Duplicates were initially removed from the search result. The remaining articles were then screened by scanning titles and abstracts for the inclusion criteria: articles studying the relationship between having the PLA2 polymorphism and risk of DVT, PE, and VTE (at least one of these); studies calculated odds ratio; and published articles in English language.

The exclusion criteria of this review study were as follows: not original articles including commentaries, review articles, case reports, and letters; papers in any language except English; articles with less than ten patients; articles that do not contain full text, including
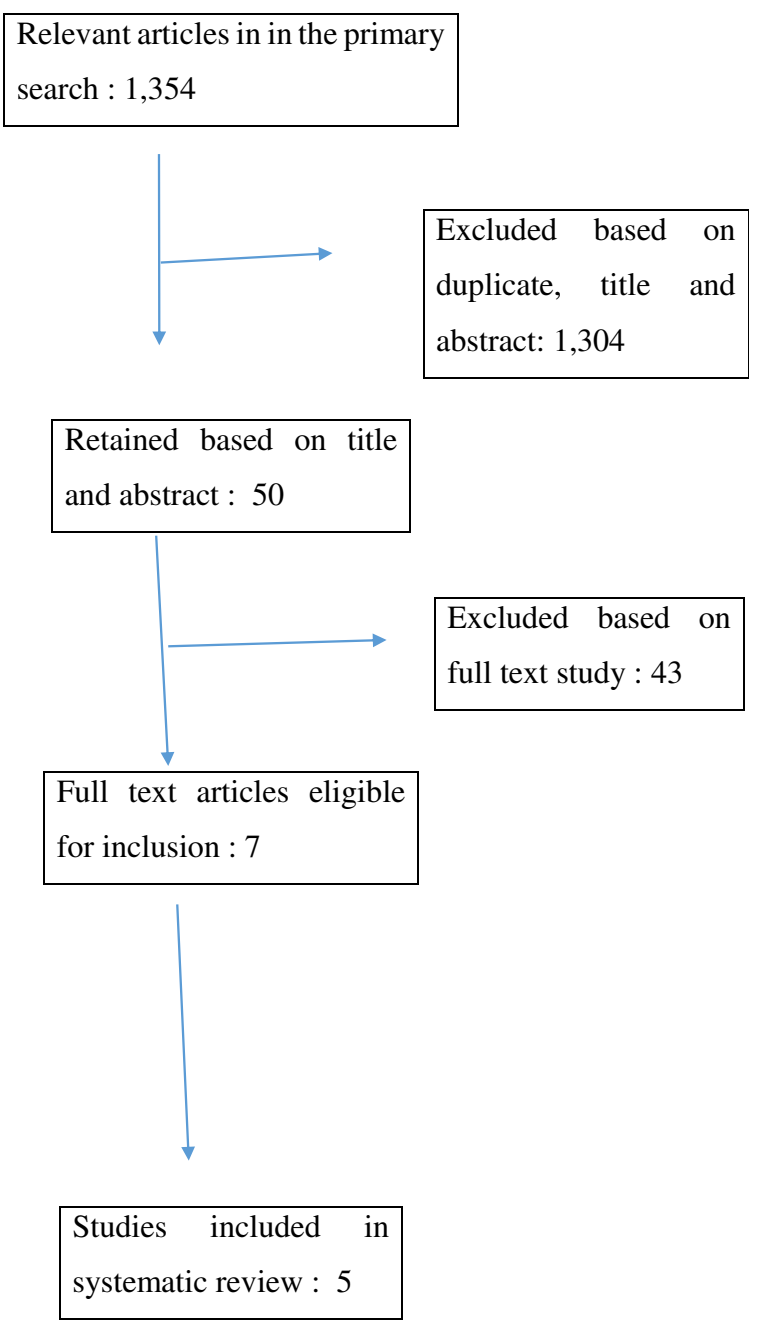

Fig. 1 PRISMA flow diagram: summary of search strategy in this study

articles presented at congresses and conferences and symposiums.

Article selection was conducted by authors independently. Any disagreement among reviewers was resolved by discussion and reached consensus finally.

\section{Data extraction and quality assessment}

One of the reviewers thoroughly investigated the article titles and abstracts to determine the competency of selected articles.

The following data were obtained from each article: first author, year of publication, study population, number of patients, patient characteristics including: age, gender, etc. and odd ratio with $95 \%$ confidence interval (Table 1).

\section{Results}

The initial search generated 1354 articles, five of which were identified as being strongly relevant following 
Table 1 Extracted data from included studies investigating the correlation between PLA2 polymorphism and VTE

\begin{tabular}{|c|c|c|c|c|c|c|c|c|}
\hline Author & Country & Patients number & Median age & OR & $95 \% \mathrm{Cl}$ & Type of disease & Frequency of PLA2 polymorphism (\%) & $p$ value \\
\hline Ridker et al 1997 & USA & 121 & 61 & 1.07 & $0.7-1.6$ & VTE & 28.1 & 0.5 \\
\hline Ivanov et al 2008 & Bulgaria & 51 & $48.7 / 45.47$ & 3.27 & $1.36-7.93$ & $P E$ & 35.3 & 0.003 \\
\hline Atzeni et al 2011 & Italy & 36 & 34 & 2.2 & $1.1-4.5$ & $\begin{array}{l}\text { Behcet's diseases } \\
\text { with DVT }\end{array}$ & 41.7 & 0.04 \\
\hline $\begin{array}{l}\text { Pourgheysari et al } \\
2012\end{array}$ & Iran & 72 & 52.12 & 3.4 & $1.8-6.44$ & VTE & 27.8 & $<0.001$ \\
\hline \multirow[t]{3}{*}{ Karimi et al 2015} & Iran & 23 & NR & 1.33 & $0.37-4.71$ & PE/DVT & 13 & NS \\
\hline & & 37 & & 1.37 & $0.5-3.82$ & $P E$ & 13.5 & \\
\hline & & 35 & & 6.65 & $3.09-14.30$ & DVT & 42.9 & $<0.001$ \\
\hline
\end{tabular}

NR data not reported, NS not significant, $N$ number, OR odds ratio, $C$ confidence interval, VTE venous thromboembolism, $P E$ pulmonary embolism, DVT deep vein thrombosis

review of title and abstract. The detailed selection process is presented in Fig. 1 and a detailed review of coherent data is collected in Table 1. The included articles were studies about the association between PLA2 polymorphism and DVT, PE, or VTE.

The patient populations of the selected papers were from various nationalities.

All patients contributing in these studies were diagnosed with venous thrombotic events with or without PE. The patient populations ranged between 23 and 121 cases.

In all evaluated studies, PLA2 polymorphism was evaluated via restricted fragment length polymorphism (RFLP) PCR.

In all studies, DVT was diagnosed and confirmed using standard methods including ultrasonography, Doppler ultrasonography, D-dimer, and clinical symptoms. Various techniques were also used for PE diagnosis such as clinical presentation, chest X-ray, ventilation perfusion lung scan, electrocardiogram, determination of blood gases, and Ddimer test. PLA2 polymorphism prevalence was reported $13-42.9 \%$ in patients (mean $=27.95 \%$ ).

The median age was within the range of 34-61 years. One of five papers compared age between cases with thrombosis risk concomitant with PLA2 allele and without it [15].

PLA2 polymorphism was associated with VTE in one study [14], but not in another one [15] (Table 1). One of the studies found no correlation between PLA2 polymorphism and thrombotic events in patients with age 60 years or older $(P=0.5)$ [15]. In two of the selected studies, the correlation between PE and PLA2 polymorphism was investigated; one of them showed no association [19], whereas the other one reported the correlation $(P=$ 0.003) [20]. In the latter, the prevalence of PLA2 in patients with recurrent PE events was 37\% (OR 3.52, CI 1.21-10.3) and one patient was homozygous genotype for this polymorphism.

The association between PLA2 polymorphism and DVT was reported in one of the articles. According to the findings, the frequency of PLA2 polymorphism was significantly higher in DVT, compared to control group $(P<0.001)$ [19]; however, no significant difference was found in the frequency of this polymorphism between $\mathrm{PE} / \mathrm{DVT}$ or PE patients and control group.

One of the studies investigated the association between PLA2 polymorphism and thrombotic events in Behcet's disease [21]. In this study, the frequency of PLA2 polymorphism was compared between the Behcet patients with DVT and control group. No significant difference was observed between Behcet's disease (BD) and control in the frequency of PLA2 polymorphism, but was found between these patients with DVT and control (OR 2, CI 1.1-3.7) (21). Carriage rate was also significantly higher in BD patients with DVT than control $(P=$ 0.044, OR 2.2, CI 1.1-4.5).

The association between recurrent events and PLA2 polymorphism was found in another study [14]. Carriage of this polymorphism had more recurrent VTE events than other patients.

In one of the studies, the effect of aspirin consumption was studied on the association between PLA2 polymorphism and thrombosis. The exclusion of these patients from the cohort had no effect on final output [15].

The prognostic value of PLA2 polymorphism and its correlation with DVT, PE, or VTE has not been thoroughly evaluated. Nevertheless, the predictive value of this genetic marker has been mostly evaluated in cases with stroke or coronary thrombosis [12, 13, 22].

In 1996, Weiss et al. reported a relationship between the PLA2 allele and elevated risk of premature coronary thrombosis [12]. Some years later, in 1999, Feng et al. suggested that PLA2 allotype in GP IIb/IIIa could be a risk factor in patients with VTE. The PLA1/PLA2 polymorphism is characterized by increased affinity of the GP IIb/IIIa platelet receptor for fibrinogen and enhances platelet aggregation as a baseline for thrombosis [22] Despite this, in 2008, Ivanov et al. declared no association between PLA2 polymorphism and risk of VTE, but 
it could be a potential risk factor for developing PE [20]. Their data showed comparable OR to other known thrombophilic mutations, FVL and FII G20210A. They reported that dual carriers of FVL/PLA2 along with early manifestation and unusual position of thrombosis had recurrent PE.

In another study in 2012, Pourgheysari et al. reported that PLA2 polymorphism is highly associated with VTE [14].This was the only inherited thrombophilic risk factor that was correlated with VTE among investigated polymorphisms, FVL, FIIG20210A and HTHFR. The PLA2 polymorphism also played a role in recurrent events in spite of the other polymorphisms. This finding demonstrated the importance of population-based studies.

According to the results of this systematic review, PLA2 polymorphism increased the thrombotic tendency, but no consistency was found between different studies. While Ivanove et al. demonstrated the association of the polymorphism with PE, Karimi et al. found no relationship. Instead, they found the association with DVT $[20,19]$.

A main limitation of the present review was the lack of enough comparable reports and paucity of research in this study. Due to the low number of publications, the study is not adequately powered to identify the importance of this polymorphism. Because of the limited accessibility of data, it was not possible to perform univariate or multivariate analyses of individual markers in all studies.

\section{Conclusion}

It was concluded that PLA2 polymorphism is a risk factor for VTE in some patients. Whether this marker can be used as an important factor in the diagnosis and treatment of patients with DVT or PE needs more investigation. The predictive value of the polymorphism is not clear from the existing publications. As the data were little and somehow incompatible, it cannot be concluded that PLA2 polymorphism is associated with an increased risk of VTE and this hypothesis needs more trainings.

\section{Abbreviations \\ BD: Behcet's disease; DVT: Deep vein thrombosis; FVL: Factor V Leiden; GP: Glycoprotein; PE: Pulmonary embolism; PLA1: Platelet antigen 1; PLA2: Platelet antigen 2; PRISMA: Preferred Reporting Items for Systematic Reviews and Meta-analyses; RFLP PCR: Restricted fragment length polymorphism PCR; VTE: Venous thromboembolism; WWF: Von Willebrand Factor}

\section{Authors' contributions}

BP: Final checking of information, participating in essay writing and submission of article. RB: Searching and gathering articles and extracting information and participating in essay writing. ZR: Searching and gathering articles and extracting information and participating in essay writing. All authors have read and approved the manuscript.

Availability of data and materials

This article is a review article and does not require any materials.
Ethics approval and consent to participate

Since the type of article is review and we have not directly studied human beings, we did not need ethics approval and consent to participate.

\section{Consent for publication}

All authors agree to publish the article.

\section{Competing interests}

The authors declare that they have no competing interests.

\section{Author details}

${ }^{1}$ Cellular and Molecular Research Center, Basic Health Sciences Institute, Shahrekord University of Medical Sciences, Shahrekord, Iran. ${ }^{2}$ School of Medicine, Tehran University of Medical Sciences, Tehran, Iran. ${ }^{3}$ Medical Plants Research Center, Basic Health Sciences Institute, Shahrekord University of Medical Sciences, Shahrekord, Iran. ${ }^{4}$ Department of Pathology and Hematology, Shahrekord University of Medical Sciences, Shahrekord, Iran.

Received: 15 August 2019 Accepted: 13 December 2019

Published online: 27 December 2019

\section{References}

1. Pinniger J, Prunty FTG (1946) Some observations on the blood-clotting mechanism. The role of fibinogen and platelets, with reference to a case of congenital afibrinogenaemia. British journal of experimental pathology 27(3):200

2. Floyd CN, Ellis BH, Ferro A (2014) The PIA1/A2 polymorphism of glycoprotein IIla as a risk factor for stroke: a systematic review and metaanalysis. PLoS One. 9(7):e100239

3. Broos K, Feys HB, De Meyer SF, Vanhoorelbeke K, Deckmyn H (2011) Platelets at work in primary hemostasis. Blood reviews. 25(4):155-167

4. Wagner CL, Mascelli M, Neblock D, Weisman H, Coller B, Jordan R (1996) Analysis of GPIIb/IIla receptor number by quantification of 7E3 binding to human platelets. Blood. 88(3):907-914

5. Bennett JS (2005) Structure and function of the platelet integrin allb $\beta 3$. Journal of Clinical Investigation. 115(12):3363

6. Calvete J (1994) Clues for understanding the structure and function of a prototypic human integrin: the platelet glycoprotein Ilb/lla complex. Thrombosis and haemostasis. 72(1):1-15

7. Newman PJ, Derbes RS, Aster RH (1989) The human platelet alloantigens, PIA1 and PLA2, are associated with a leucine33/proline33 amino acid polymorphism in membrane glycoprotein IIla, and are distinguishable by DNA typing. Journal of Clinical Investigation. 83(5):1778

8. Michelson AD, Furman MI, Goldschmidt-Clermont P, Mascelli MA, Hendrix C, Coleman L et al (2000) Platelet GP IIla PI A polymorphisms display different sensitivities to agonists. Circulation. 101(9):1013-1018

9. Kujovich $J \mathrm{~L}(2011)$ Factor $\vee$ Leiden thrombophilia. Genetics in Medicine. 13(1):1-16

10. Marchiori A, Mosena L, Prins MH, Prandoni $P$ (2007) The risk of recurrent venous thromboembolism among heterozygous carriers of factor $V$ Leiden or prothrombin G20210A mutation. A systematic review of prospective studies. Haematologica. 92(8):1107-1114

11. Pourgheysari B, Drees F, Hashemzadeh-Chaleshtori M (2014) Factor XIIIAV34L and factor XIIIB-H95R in venous thromboembolism in central Iran: protective and neutral. Blood Coagulation \& Fibrinolysis. 25(5):439-443

12. Weiss EJ, Bray PF, Tayback M, Schulman SP, Kickler TS, Becker LC et al (1996) A polymorphism of a platelet glycoprotein receptor as an inherited risk factor for coronary thrombosis. New England Journal of Medicine. 334(17): 1090-1094

13. Gurbel PA, Bliden KP, Cohen E, Navickas IA, Singla A, Antonino MJ et al (2008) Race and sex differences in thrombogenicity: risk of ischemic events following coronary stenting. Blood Coagulation \& Fibrinolysis. 19(4):268-275

14. Pourgheysari B, Boroujeni HR, Hasheminia AM, Drees F (2013) PLA2 polymorphism of platelet glycoprotein Ilb/llla but not Factor $V$ Leiden and prothrombin G20210A polymorphisms is associated with venous thromboembolism and more recurrent events in central Iran. Blood Coagulation \& Fibrinolysis. 24(5):471-476

15. Ridker PM, Hennekens CH, Schmitz C, Stampfer MJ, Lindpaintner K (1997) PIA1/A2 polymorphism of platelet glycoprotein IIla and risks of myocardial infarction, stroke, and venous thrombosis. The lancet. 349(9049):385-388 
16. Maynard G, Stein J (2010) Designing and implementing effective venous thromboembolism prevention protocols: lessons from collaborative efforts. Journal of thrombosis and thrombolysis. 29(2):159-166

17. Goldhaber SZ, Visani L, De Rosa M (1999) Acute pulmonary embolism: clinical outcomes in the International Cooperative Pulmonary Embolism Registry (ICOPER). The Lancet. 353(9162):1386-1389

18. Geerts WH, Bergqvist D, Pineo GF, Heit JA, Samama CM, Lassen MR et al (2008) Prevention of venous thromboembolism: American College of Chest Physicians evidence-based clinical practice guidelines. Chest journal 133(6_ suppl):381S-453S

19. Karimi A, Abolhasani M, Hashemzadeh-Chaleshtori M, Pourgheysari B (2015) A preliminary study of inherited thrombophilic risk factors in different clinical manifestations of venous thromboembolism in central Iran. The Indian journal of medical research. 142(1):46

20. Ivanov P, Komsa-Penkova R, Kovacheva K, Ivanov Y, Stoyanova A, Ivanov I et al (2008) Impact of thrombophilic genetic factors on pulmonary embolism: early onset and recurrent incidences. Lung. 186(1):27-36

21. Atzeni F, Boiardi L, Nicoli D, Farnetti E, Casali B, Sarzi-Puttini P et al (2011) PLA1/A2 polymorphism of the platelet glycoprotein receptors IIIA in Behçet's disease. Clin Exp Rheumatol. 29(4 Suppl 67):S38-S43

22. Feng $D$, Lindpaintner $K$, Larson MG, Rao VS, O'Donnell CJ, Lipinska I et al (1999) Increased platelet aggregability associated with platelet GPIIla PI A2 polymorphism. Arteriosclerosis, thrombosis, and vascular biology. 19(4): 1142-1147

\section{Publisher's Note}

Springer Nature remains neutral with regard to jurisdictional claims in published maps and institutional affiliations.

\section{Submit your manuscript to a SpringerOpen ${ }^{\circ}$ journal and benefit from:}

- Convenient online submission

- Rigorous peer review

- Open access: articles freely available online

- High visibility within the field

- Retaining the copyright to your article 